Cahiers « Mondes anciens »

ANCIENS

Histoire et anthropologie des mondes anciens

$10 \mid 2018$

Les politiques familiales dans les mondes antiques

\title{
Entre-soi matrimonial et construction communautaire
}

La question des mariages « mixtes » en Méditerranée orientale à l'époque classique

Endogamy and Community Building: The Ban of Mixed Marriages in the Classical Eastern Mediterranean Societies

Jérôme Wilgaux

\section{OpenEdition \\ Journals}

Édition électronique

URL : http://journals.openedition.org/mondesanciens/1974

DOI : 10.4000/mondesanciens. 1974

ISSN : 2107-0199

Éditeur

UMR 8210 Anthropologie et Histoire des Mondes Antiques

Référence électronique

Jérôme Wilgaux, "Entre-soi matrimonial et construction communautaire », Cahiers " Mondes anciens » [En ligne], 10 | 2018, mis en ligne le 07 février 2018, consulté le 21 avril 2019. URL : http:// journals.openedition.org/mondesanciens/1974 ; DOI : 10.4000/mondesanciens.1974

Ce document a été généré automatiquement le 21 avril 2019.

\section{c) (i) (9)}

Les Cahiers "Mondes Anciens » sont mis à disposition selon les termes de la licence Creative Commons Attribution - Pas d'Utilisation Commerciale - Pas de Modification 4.0 International. 


\title{
Entre-soi matrimonial et construction communautaire
}

\author{
La question des mariages « mixtes » en Méditerranée orientale à \\ l'époque classique \\ Endogamy and Community Building: The Ban of Mixed Marriages in the \\ Classical Eastern Mediterranean Societies
}

Jérôme Wilgaux

$1 \quad$ L'un des enjeux majeurs des études grecques est bien sûr de comprendre les modalités selon lesquelles les communautés civiques - les poleis - se sont construites, la manière dont elles ont progressivement imposé leurs institutions, leurs valeurs, leurs propres catégorisations de la société, et ainsi édifié un ordre qui dans ses multiples dimensions (politique, social, économique, religieux...) leur est spécifique.

Depuis les travaux, au xix ${ }^{\mathrm{e}}$ siècle, de George Grote, de Numa-Denis Fustel de Coulanges, ou bien encore des évolutionnistes tels que Henry Sumner Maine et Lewis Morgan, jusqu'aux recherches les plus récentes, le rôle qu'ont pu jouer les relations et groupes de parenté au cours de ce processus, dans la construction des liens communautaires comme dans leur définition, n'a cessé d'être questionné. C'est ainsi par quelques remarques générales sur l'articulation complexe du parental et du politique dans le monde grec des cités, et sur la réglementation nécessairement conjointe de la citoyenneté et de la parenté, que commencera cette étude ; dès lors, le dossier documentaire grec le plus complet est sans nul doute celui constitué par la loi sur la citoyenneté de Périclès, adoptée en 451-450 par l'assemblée athénienne. Les multiples études que cette loi a suscité, bien souvent limitées au seul cas athénien alors que la double filiation se retrouve dans d'autres cités, nous invitent cependant aujourd'hui à essayer d'adopter une perspective plus ample, comparative, et même à nous intéresser aux réglementations matrimoniales observées dans d'autres régions de la Méditerranée classique. L'exercice est assurément difficile et incertain, du fait des discontinuités inhérentes à notre documentation, mais au-delà du seul monde grec, il nous a paru intéressant d'élargir notre enquête à la société judéenne des $V^{e}$ et $I^{e}$ siècles av. J.-C. En effet, de nombreuses études portant sur cette société (cf. 
notamment Halpern 2004, Fröhlich 2005, Eskenazi 2006, Fried 2009 et 2015) ont, ces dernières années, adopté une telle démarche et confronté deux évolutions semblant présenter des traits communs, à savoir l'interdiction des mariages mixtes en Judée au cours de cette période, et la loi de Périclès imposant pour sa part la double ascendance pour la transmission de la citoyenneté. Au-delà des différences manifestes entre ces deux systèmes socio-politiques, selon ces recherches, la démarche comparative pourrait donc permettre aux historiens d'affiner leurs interprétations. Dans le cadre de cet article, notre ambition ne peut être que limitée, même si nos remarques se veulent synthétiques : sans entrer dans le détail des interprétations proposées par les spécialistes du monde judéen, ce sont avant tout deux traditions historiographiques que nous comparons, en ne retenant que les points qui nous ont paru les plus suggestifs et en développant dès lors nos propres interprétations ${ }^{1}$. En arrière-plan de cette brève présentation et discussion de ces cas particuliers et de ce dossier historiographique, se pose la question de savoir s'il est possible de retrouver des logiques sociales communes au sein de l'espace méditerranéen, appréhendé en tant qu' "aire du mariage dans un degré rapproché », comme nous y invitent des anthropologues (cf. e.g. Bonte 1994 ; Bonte et al., 2011).

\section{Parenté et citoyenneté dans le monde grec : quelques remarques générales}

3 Les critères de citoyenneté ont bien sûr pu être très divers dans le monde grec antique, et prendre en compte par exemple les patrimoines, les activités économiques, les modes de vie et les modes de participation à la défense du territoire; mais la naissance - le fait d'être né d'un membre ou de deux membres de la communauté civique, et de voir cette naissance reconnue comme légitime -, a toujours constitué un critère essentiel, et bien souvent nos sources nous montrent que la famille et les relations de parenté ont été perçues comme constituant les fondements mêmes de la communauté civique (pensons par ex. au livre I des Politiques d'Aristote, ou bien au début de l'Économique (I, 1) attribuée au même philosophe : « Une cité est un ensemble de maisons, de terres et de richesses qui puisse se suffire à elle-même pour y bien vivre. »). De manière générale, nous pouvons dire que la cité a pu se percevoir comme un rassemblement de familles, d'oikoi, ou comme un groupe de frères descendant de mêmes ancêtres (cf. Brulé 2007, Wilgaux 2011), et cette parenté commune créait des privilèges et des obligations, des droits et des devoirs.

4 Mais dans le même temps, il nous faut également constater que cette parenté n'a cessé d'être construite par la cité. Nos sources nous donnent ainsi de multiples exemples de réorganisation des cadres civiques, avec la création de nouvelles tribus ou d'autres subdivisions (réunissant alors des membres d'origines diverses), mais qui se présentent à nous pour autant comme des groupes de parenté (cf. Roussel 1976). L'un des exemples les plus évidents est sans doute celui, à Athènes, des dèmes clisthéniens, une circonscription territoriale dont l'appartenance est en fait héréditaire, en l'occurrence patrilinéaire ; un groupe initialement défini par le lieu de résidence, lors des réformes de Clisthène en 508-507, peut être ainsi appréhendé comme un groupe de filiation, lui-même subdivision d'une des composantes majeures de la cité, au recrutement également parental, la tribu.

5 Dès lors, si, dans une première approche, il est possible de dire qu'une cité est un rassemblement de personnes parentes entre elles, et donc que c'est parce qu'on appartient à un groupe de parenté que l'on est citoyen, en fait, d'un autre point de vue, 
c'est parce qu'on est reconnu comme citoyen que l'on appartient à un groupe de parenté . Dans cette perspective, légiférer sur les modes d'affiliation à la cité, c'est nécessairement légiférer sur la parenté des citoyens, c'est redéfinir leur parenté. De fait, dans ce monde grec des cités, de manière générale, tout individu qui perd sa citoyenneté sort concrètement de sa parenté d'un point de vue juridique.

6 Cette association étroite du parental et du politique se manifeste pleinement dans le dossier qui focalise toutes les attentions des hellénistes lorsqu'ils s'intéressent à la manière dont la parenté entre en ligne de compte dans la définition de la citoyenneté, à savoir celui de la loi de Périclès de 451-450: puisqu'à partir de cette loi, tout enfant né à Athènes d'un parent non athénien, quelles que soient les modalités d'union des parents en question, ne peut plus être citoyen athénien, il ne peut donc plus accéder aux prérogatives civiques, à ces multiples droits dont bénéficient les citoyens (dont le droit d' enktêsis notamment, c'est-à-dire le droit de propriété), et à ce titre il ne peut plus prétendre à hériter du patrimoine de ses parents. C'est bien la notion même de notheia (bâtardise) qui a été ainsi redéfinie par la Loi de Périclès.

7 La cité utilise donc le langage de la parenté pour définir son identité et sa composition, pour exprimer sa solidarité, pour se structurer et pour penser son mode d'organisation, mais dans le même temps elle ne cesse de manipuler son organisation parentale pour imposer ses propres logiques et donc ses propres définitions de la parenté, redéfinissant les catégories mises en œuvre et les pratiques prescrites, légitimes. De ce point de vue, filiation et alliance vont assurément de pair. Toute modification des règles d'affiliation a des implications matrimoniales, et réciproquement. Là encore, les travaux, fort nombreux, portant sur la loi de Périclès de 451 ont bien sûr montré cette interdépendance de la définition de la citoyenneté et des normes et pratiques filiatives et matrimoniales, puisqu'à partir du moment où la cité opte pour la double ascendance pour la transmission de sa citoyenneté, elle se construit comme une communauté matrimoniale, refermée sur elle-même, une communauté dont les membres reconnus comme légitimes (gnêsioi) en ce sens qu'ils sont nés de deux parents athéniens, peuvent circuler, par l'adoption, d'un oikos à l'autre, et doivent nécessairement s'épouser entre eux pour perpétuer leurs oikoi comme la cité dans son ensemble. Si, à Athènes, les «mariages mixtes» (i.e., en l'occurrence, avec un partenaire extérieur à la communauté civique) ne semblent avoir été explicitement interdits que dans les premières décennies $\mathrm{du} \mathrm{IV}^{\mathrm{e}}$ siècle, dans les années qui suivirent le rétablissement de la démocratie de 403 (cf. Bonnard et al. 2017, p. 263), dès 451 de tels mariages ne pouvaient que placer les personnes concernées en marge ou en porte-à-faux à l'égard de la communauté civique.

8 Cette loi de Périclès a donc suscité et suscitent encore et toujours, année après année, de très nombreux travaux (voir récemment Carawan 2008, Blok 2009, Coşkun 2013 et 2014), s'interrogeant sur sa chronologie, ses motivations, son application, ses amendements possibles. Mais au-delà du seul cas athénien, qu'en était-il de manière plus générale dans l'ensemble du monde grec des cités? Des informations très générales mais cependant essentielles nous sont apportées par Aristote à ce propos. Ainsi, dans les Politiques, ce dernier nous présente le critère de la double ascendance, d'une part comme le choix le plus fréquemment constaté ${ }^{3}$, et d'autre part comme le plus légitime : le choix de critères plus restrictifs, lorsque la double ascendance est demandée sur plusieurs générations, pose en effet la question de la manière dont ont été définis les premiers citoyens ; quant au choix de critères moins contraignants, il nous est présenté soit comme une dérogation momentanée à la règle afin de pallier un manque de citoyens (III, 5, 1278a), soit comme 
une mesure excessive prise par les démocrates afin d'augmenter et de se concilier le dêmos (VI, 4, 1319b8-9).

Ces remarques générales formulées par Aristote témoignent également de la variété des situations, certaines cités pouvant octroyer la citoyenneté aux nothoi (ce qu'Aristote entend précisément par ce terme reste sujet à débat) et aux enfants nés d'un seul parent membre de la communauté civique, l'autre parent, père ou mère, pouvant être un ou une esclave, un étranger ou une étrangère.

10 Notre documentation sur ce sujet reste aujourd'hui limitée et difficile à interpréter, mais la tendance générale qui semble se dégager de nos sources est cependant bien celle d'une généralisation de la règle de la double ascendance à partir du $\mathrm{IV}^{\mathrm{e}} \mathrm{s}$. et au cours de l'époque hellénistique, avec notamment les cas de Byzance, Thasos, Rhodes, Cos, Milet, Ténos, Délos, Halicarnasse, Marseille, Alexandrie (Égypte) ou bien encore Orchomène ${ }^{4} .$.

11 Dès lors, malgré les faiblesses évidentes de notre documentation, il faut bien remarquer que les réflexions anciennes et actuelles, très nombreuses et souvent très ingénieuses, sur la loi de Périclès paraissent fort réductrices pour au moins quatre raisons :

- Ces réflexions se focalisent sur Athènes alors que cette règle de la double ascendance paraît avoir été choisie par un grand nombre de cités, au moins à partir du IV $\mathrm{s}$. (c'est du moins alors, et tout au long de l'époque hellénistique, que ces normes civiques deviennent visibles dans nos sources). Au-delà du cas athénien, c'est donc aussi cette tendance générale qui doit être expliquée (et dans le même temps la grande variété des situations).

- Elles opposent le plus souvent à la double ascendance la seule ascendance par le père, alors que nos sources nous apportent de nombreux témoignages du fait que dans un certain nombre de cités la mère seule pouvait elle aussi, tout autant que le père, transmettre la citoyennetés.

- Elles interprètent la règle de la double ascendance avant tout comme un refus de mariage avec des étrangers, des étrangères, alors que se pose tout autant la question de savoir si un mariage avec des non libres (esclaves marchandises ou esclaves du type hilotique) pouvait ou non permettre de transmettre la citoyenneté aux enfants nés de cet autre type d'union mixte (cf. Walters 1983 ; insistons bien sur ce point puisqu'à Gortyne par exemple, les mariages entre libres et esclaves sont possibles, ce qu'ils ne sont pas bien sûr à Athènes). Il faut ainsi tenir compte d'au moins deux manières de concevoir le mariage mixte dans le monde grec, c'est-à-dire prendre en considération les différences d'origine et les différences de statut, les deux pouvant bien sûr se cumuler.

- Enfin, ces questions conjointes de la double ascendance et de la possibilité ou non de "mariages mixtes" sont indissociables des modes de construction communautaire définissant le mariage légitime comme un droit propre aux membres de la communauté : des termes tels que epigamia, conubium, désignent alors ce droit civique, dont la possession est nécessaire pour donner naissance à des enfants pleinement légitimes au sein de la communauté. Si l'imposition de la double ascendance, l'interdiction des « mariages mixtes » et la définition du mariage légitime comme un droit civique relèvent d'une même logique, leur application peut bien sûr présenter de fortes différences, comme le montre clairement l'exemple romain (cf. Bonnard et al. 2017).

12 Dans le cadre de cet article, nous n'allons pas aborder bien sûr tous ces aspects, mais essayer de montrer l'intérêt d'une approche plus globale de la question, en comparant les données grecques au cas judéen. 


\section{Le choix d'une approche comparative : le parallèle avec la Judée}

13 Au-delà du seul cas athénien donc, il nous paraît intéressant d'adopter une démarche comparative pour analyser comment, dans la moyenne et longue durée, les cités grecques, mais aussi les autres sociétés méditerranéennes, quelle que soit leur organisation sociopolitique, ont pu varier dans leurs manières de définir les appartenances et dans leur acceptation ou non des mariages mixtes, tels que nous venons de les définir ${ }^{6}$.

14 De ce point de vue, notre attention s'est portée sur un dossier très particulier, là encore marqué par une inflation considérable de publications au cours des dernières décennies, le cas de la Judée des $\mathrm{VI}^{\mathrm{e}}$-IV ${ }^{\mathrm{e}} \mathrm{s}$., c'est-à-dire lors de la période d'hégémonie perse sur ce territoire et sur cette communauté.

Ce détour par la Judée peut paraître à première vue surprenant, tant les contextes sont différents, mais la question du mariage avec les étrangers occupe une place essentielle dans les sources relatives à la période d'occupation perse, à savoir les Livres d'Esdras et de Néhémie, et il nous a paru donc intéressant, en tant qu'helléniste, de prendre en compte ce que nous disent les sources à ce propos et la manière dont les chercheurs aujourd'hui interprètent la documentation et expliquent ce refus des mariages mixtes.

Le véritable point de départ de cette étude a en fait été le constat que plusieurs études récemment consacrées à ce cas judéen ont précisément adopté cette démarche comparative et donc proposé une analyse globale des cas judéen et athénien (cf. supra). Dans le cadre limité de cet article, nous n'en proposerons que quelques grandes lignes.

Résumons la situation.

Si nous suivons ces sources essentielles que sont les Livres d'Esdras et de Néhémie, les conquêtes de Cyrus et l'avènement de l'Empire perse permirent le retour en Judée d'une partie des exilés du début $d u \mathrm{VI}^{\mathrm{e}} \mathrm{s}$. et ouvrirent ainsi une période de restauration culturelle et religieuse, marquée par la construction d'un nouveau temple et une application qui se voulait plus scrupuleuse que par le passé des enseignements et prescriptions du Pentateuque.

19 Dans les années 450-440 (selon la chronologie la plus souvent retenue, mais qui peut être contestée, l'activité d'Esdras pouvant être notamment datée de 398), Esdras et Néhémie auraient joué un rôle crucial dans cette réorganisation communautaire et ces réformes religieuses : ils auraient notamment imposé l'interdiction des mariages mixtes, obligeant les Judéens concernés à se séparer de leurs épouses étrangères (et des enfants nés de ces unions) $)^{7}$.

La période perse marquerait ainsi une évolution notable des prohibitions matrimoniales à l'égard des étrangers: si jusqu'alors, les interdits matrimoniaux ne concernaient que certaines populations précisément définies, telles que les Cananéens, les Moabites, les Ammonites, l'interdit imposé par Esdras et Néhémie porterait désormais sur l'ensemble des populations non judéennes; d'autre part, si le Pentateuque condamne avant tout les mariages mixtes en ce qu'ils pourraient détourner les Judéens de leurs traditions religieuses et ainsi les détacher de l'alliance conclue avec leur dieu Yahvé, à partir du Livre d'Esdras, la rupture de l'alliance provoquée par ces mariages est d'abord expliquée 
par la souillure que représente tout mélange entre le «peuple saint», la "semence sainte », et les autres populations.

21 Cette "rhétorique de la pureté » est essentielle, nous y reviendrons, mais retenons simplement ici l'idée que cette condamnation des mariages mixtes aboutit à une dichotomie claire entre Judéens et non Judéens, les Judéens devant préserver la singularité, l'exceptionnalité, de leur peuple en se mariant entre eux. Dans un contexte marqué par la domination sans partage du pouvoir perse sur un empire multi-ethnique, et alors que l'ethnoclasse dominante perse (pour reprendre cette expression à Pierre Briant 1996) non seulement pratique l'intermariage mais a pu le considérer comme un instrument de consolidation de son hégémonie, le peuple judéen afficherait donc sa différence, entre autres, par ses choix matrimoniaux.

De longue date, des commentateurs ont pu remarquer des similitudes, ne serait-ce que chronologique, entre la loi de Périclès et les interdits énoncés par Esdras et Néhémie, et de nombreuses études récentes se sont donc proposées de rendre compte globalement de ces évolutions.

Il convient de ne pas oublier que ces similitudes peuvent provenir non pas tant des faits eux-mêmes que du contexte d'écriture de nos sources : les Livres d'Esdras et de Néhémie, tout comme les autres composantes de la Bible, posent de difficiles problèmes de datation, ces écrits pouvant avoir été composés plus tardivement que ne le laisserait penser leur contenu, ou avoir été l'objet de réécritures déplaçant dans le passé, de manière anachronique donc, des problèmes qui ne se posaient pas en ces termes à l'époque perse (voir notamment Grabbe 2004). Ces sources peuvent donc avoir été contaminées, à l'époque hellénistique, par la connaissance que leurs rédacteurs pouvaient avoir du monde grec, de son histoire et de sa culture.

Quoi qu'il en soit de la datation des textes, les historiens sont confrontés à des sources, des réformes, des actions politiques, qui dans des contextes différents valorisent la double ascendance et s'opposent en conséquence, explicitement ou implicitement, aux mariages avec des membres extérieurs à la communauté. Comment en rendre compte?

Il ne s'agit pas ici de passer en revue l'ensemble des interprétations développées par les spécialistes des textes bibliques (cf. notamment Fried 2009 et 2015 ; Eskenazi 2006), mais de proposer nos propres réactions, en tant qu'helléniste, plus familier des interprétations de la loi de Périclès. Est-il possible de dégager, en étudiant les évolutions de ces deux sociétés, un modèle commun de construction communautaire? Quels sont du moins les principaux points communs qui nous paraissent devoir être mis en évidence ${ }^{8}$ ?

\section{Filiation et alliance au fondement du « commun »}

De manière générale, dans le cas judéen comme dans le cas athénien, les interprétations actuelles insistent sur le caractère identitaire de ces réformes et sur le double mouvement, d'exclusion et d'inclusion, qu'elles engendrent: c'est dans une même dynamique qu'une communauté s'auto-définit, en se distinguant des autres populations et en renforçant son homogénéité, sa cohésion; un double mouvement où les traits qui distinguent radicalement de l'autre sont aussi ceux qui font des membres de la communauté des semblables, permettant ainsi de "construire du commun», pour reprendre cette expression fort utilisée aujourd'hui. 

joua une rhétorique de la pureté (le refus de tout mélange) et de la bonne naissance. Les analyses de Josine Blok (2009) prennent ainsi pour point de départ l'idée que la citoyenneté se définissait avant tout par la participation aux mêmes cultes, aux mêmes rituels, une participation destinée à perpétuer les liens tissés avec les dieux ancestraux de la communauté. Dans cette perspective, la loi de Périclès aurait construit la communauté civique athénienne sur le modèle des genê (lignages) aristocratiques athéniens qui pouvaient revendiquer l'ancienneté et la qualité de leur origine, et à ce titre conservaient la mainmise sur des prêtrises héréditaires. Toujours selon Josine Blok, la loi de Périclès témoigne ainsi que désormais l'eugeneia (bonne naissance) des plus anciens lignages était conçue comme partagée par l'ensemble des Athéniens, mais pour être préservée demandait donc que la citoyenneté soit exclusivement réservée aux enfants nés de deux parents eux-mêmes porteurs de cette eugeneia, donc membres de la communauté civique.

C'est un processus comparable qui peut être constaté dans le cas judéen : dès le Lévitique (23:7-15), les prêtres avaient été astreints à des choix matrimoniaux plus exigeants et notamment à ne pas épouser des étrangères, afin de préserver leur pureté et leur proximité avec le divin. Désormais, avec Esdras et Néhémie, cette rhétorique de la pureté s'étend à l'ensemble du peuple judéen tandis que les prêtres se voient imposer des choix encore plus sélectifs (cf. Fried 2015).

Ce parallèle est évidemment très suggestif, et nous le poursuivrons par quelques remarques. Les modes de construction communautaire (quelle que soit l'ampleur des communautés concernées), telles qu'elles nous sont présentées dans ces analyses, associent donc aux liens politiques les solidarités parentales et religieuses. En fait, dans le monde grec archaïque ou classique, plusieurs cas sont bien connus de l'adoption de normes endogamiques par une catégorie particulière de la population, citons par exemple les Bacchiades à Corinthe du milieu du viII ${ }^{\mathrm{e}} \mathrm{s}$. au milieu du viI ${ }^{\mathrm{e}} \mathrm{s}$. (Hérodote, L'Enquête, V, 92) ou bien les Héraclides à Sparte (et donc les deux familles royales spartiates), puisque Plutarque nous apprend qu'il était interdit à ces derniers d'engendrer une descendance avec une épouse d'origine étrangère (Plutarque, Agis et Cléomène, 11, 2) : dans les cas des Bacchiades et des Héraclides, cette norme endogamique peut aisément s'expliquer par la volonté d'une part de fixer des frontières étanches au groupe concerné et d'autre part, en conséquence, de préserver au sein de ce groupe l'ensemble des privilèges dont il bénéficie ; cette même ambition peut être attribuée à l'endogamie civique.

Mais comment qualifier précisément ce processus? Dire que le mariage devient endogame signifie qu'il devient restreint aux membres de la communauté; ou pour le dire autrement il est désormais construit comme un "universel» au sein de cette communauté, c'est-à-dire comme participant de la fabrication ou du renforcement d'une identité commune : un ensemble de qualités, de normes et de pratiques qui étaient propre à une catégorie restreinte de la population (la bonne naissance, la pureté des origines, l'endogamie) devient la propriété, la caractéristique de l'ensemble des membres de la communauté. Ce processus d'universalisation, de généralisation, de qualités, de normes, de pratiques est indissociable de représentations partagées du corps et de l'engendrement qui «naturalisent » les différences et font des descendants des ancêtres fondateurs les seuls appropriés "naturellement » à bénéficier de certains privilèges, à développer des relations qui leur sont spécifiques (et qui sont exigés d'eux), ne serait-ce qu'avec le divin.

Cahiers « Mondes anciens », $10 \mid 2018$ 
31 De ce point de vue, cela nous permet de rappeler que la parenté ne consiste pas simplement en des normes et des pratiques, mais est un langage, une idéologie pour penser la société et la légitimer ; à ce titre, la parenté est bien un instrument de mise en ordre du social, d'agrégation, de hiérarchisation et d'exclusion des individus ; elle est un moyen certes de construire du commun mais en même temps de fonder en nature des distinctions radicales - statutaires - au sein de la société, les appartenances définies étant exclusives les unes des autres (cf. notamment les analyses développées par Olyan 2000, présentées ci-dessous).

Si les élites, religieuses ou politiques, ont pu adopter des normes endogamiques (généralisées donc à l'époque classique à l'ensemble des membres de la communauté), elles ont pu également, au contraire, privilégier les mariages lointains et nouer ainsi des alliances avec des partenaires extérieurs à leur communauté d'origine mais semblables dans leurs positions sociales et politiques. La double ascendance ayant pour conséquence d'exclure des choix matrimoniaux ces étrangers, qu'ils soient ou non résidents, l'une des motivations possibles de cette nouvelle exigence - c'est du moins une explication que nous retrouvons aujourd'hui développée par les chercheurs dans l'un et l'autre dossier, judéen comme athénien - pouvait donc être la volonté de s'opposer aux comportements des élites, pour lesquelles les mariages permettaient de tisser des réseaux sociaux, politiques au-delà des frontières communautaires.

Est-ce là l'une des motivations premières de ces réformes, les mariages mixtes constituaient-ils une pratique particulièrement attribuée aux élites? ces points restent incertains, mais ce qui frappe en comparant les évolutions de ces deux sociétés dans la moyenne et longue durée, disons entre le $\mathrm{VII}^{\mathrm{e}}$ et le $\mathrm{V}^{\mathrm{e}} \mathrm{s}$., pour autant que nous puissions les reconstituer de manière fiable, est que ce rejet des mariages mixtes semble s'inscrire dans une réorganisation plus globale, une réorganisation progressive bien entendu, des institutions familiales, caractérisée par un encadrement juridique et collectif plus strict des pratiques familiales et matrimoniales, la diffusion de la monogamie, une exclusion plus accentuée des bâtards (une distinction plus radicale entre enfants légitimes et illégitimes), l'importance désormais prépondérante de la dot au sein des prestations matrimoniales, et une valorisation des mariages entre proches, dans un cadre familial, en particulier en présence de filles sans frère ; cette valorisation de l'endogamie est donc tout à la fois familiale et communautaire.

Dans le cas athénien (cf. par ex. Leduc 1990, Lape 2002-2003), ces évolutions sont antérieures à la loi de Périclès et sont généralement associées aux réformes de Solon. Dans le cas judéen, la chronologie est beaucoup moins claire et les pratiques sont beaucoup plus diversifiées, mais les points essentiels que nous venons d'évoquer peuvent être également observés au cours de la période envisagée, comme le montrent notamment les travaux de M. L. Satlow (2001) et T. M. Lemos (2010) ${ }^{9}$.

L'impression qui se dégage de ces deux sociétés est donc que le choix de restreindre le mariage aux seuls membres de la communauté participe de la mise en place d'un nouveau modèle social et s'inscrit notamment dans une tendance générale à la valorisation tout à la fois du couple conjugal (monogamie) et de la bilatéralité des transmissions et des appartenances, ce couple conjugal étant perçu comme la réunion de deux familles traitées davantage que par le passé à parts égales (l'épouse, dotée, transmettant à ses enfants une partie du patrimoine de sa propre famille). 
$$
\text { pouvons ainsi distinguer en Judée, d'une }
$$
communauté, ceux qui par définition peuvent prétendre à une inscription légitime dans les structures parentales reconnues, définissent leur identité en fonction de leur position dans ces structures parentales, et en tant que tels bénéficient de privilèges (la possession conjugal et renforcement de la bilatéralité des transmissions et des appartenances, participent de la même dynamique, et ce qui apparaît clairement dans le cas athénien (nous ne nous prononcerons pas pour le cas judéen) est que cette évolution s'accompagne d'une distinction plus radicale que par le passé du mariage légitime et du concubinat, le mariage légitime devenant le mode d'union attendu entre deux Athéniens (ce qui peut s'analyser de nouveau en terme d'universalisation du mariage, désormais reconnu comme une prérogative civique), le concubinat devenant pour sa part le mode d'union attendu des personnes d'origines ou de statuts différents, par ex. entre un citoyen et une étrangère (que cette dernière soit ou non métèque à Athènes).

Pour analyser de manière globale ces évolutions, nous ne pouvons pas cependant nous en tenir à la seule exclusion des étrangers des choix matrimoniaux, et il convient de prendre en compte le cas des esclaves. De nouveau, le cas athénien est le plus clair.

Les réformes de Solon interdisant l'esclavage pour dette impliquent une origine désormais extérieure des esclaves; si nous en croyons les lois qui à l'époque classique nous sont présentées comme des lois soloniennes, les esclaves se virent également exclus d'activités considérées comme propres aux hommes libres, à savoir la fréquentation des gymnases et des palestres, les relations pédérastiques (Mactoux 1988). À la différence des dépendants lacédémoniens ou crétois par ex., qui se voyaient reconnaître une vie familiale et des formes d'union matrimoniale, les esclaves-marchandises athéniens se virent refuser toute capacité juridique, et en conséquence toute capacité matrimoniale ou filiative. À partir de quel moment devint-il impossible, à Athènes, d'engendrer un enfant légitime en s'unissant avec un(e) esclave, nos sources ne nous permettent pas de le préciser avec certitude, ce qui est clair néanmoins est que la loi de Périclès entérine cette incapacité matrimoniale des esclaves.

Qu'en est-il dans le cas judéen? Si les sources sont encore plus confuses et les évolutions moins nettes, il est frappant cependant de constater que le Livre de Néhémie condamne l'esclavage pour dettes et réprouve ainsi l'idée qu'un Judéen puisse devenir l'esclave d'un autre Judéen. Dès lors, la condamnation des mariages mixtes concerne également les esclaves (cf. Lemaire 2015).

Replacés dans la longue durée, la double ascendance et le rejet des mariages mixtes s'inscrivent donc tout à la fois dans un processus de construction identitaire, dans une réorganisation plus générale des normes familiales et matrimoniales propres aux membres de la communauté, et en tant que tels participent de cet autre processus qui aboutit à la division de la société en statuts bien différenciés, définis en termes de droits et de devoirs, de capacités et d'incapacités, les différences statutaires étant fondées sur des différences d'origine et sur une gradation de capacités dans l'ordre de la parenté, de la filiation et de l'alliance.

Résumons cela en disant que cette construction est tout à la fois ethnique et statutaire, les clivages parentaux et statutaires se cumulant.

Si nous adoptons les analyses développées notamment par Saul M. Olyan (2000), nous pouvons ainsi distinguer en Judée, d'une part, les membres de plein droit de la 
de la terre notamment); et d'autre part, ceux qui, étant exclus de ces structures parentales, sont liés à ce groupe privilégié par d'autres types de relation révélateurs de leur soumission, à savoir des relations de clientélisme (résidents libres, dépendants) ou d'asservissement (esclaves marchandises).

Chaque statut, défini ainsi en termes de droits et de devoirs, implique des rapports sociaux différents: les Judéens étaient unis par des relations égalitaires de parenté (filiation et alliance) auxquelles ils pouvaient seuls prétendre, tandis que le reste de la population leur était attaché par des rapports de subordination de diverses natures: l'évolution des normes matrimoniales permet ainsi la reproduction de ces catégories statutaires.

La distinction radicale qui s'opère à Athènes, à partir du début du $\mathrm{vI}^{\mathrm{e}} \mathrm{s}$., entre membres de la communauté et esclaves, puis à partir $\mathrm{du} \mathrm{v}^{\mathrm{e}} \mathrm{s}$., entre citoyens et métèques, témoigne là aussi de cette catégorisation de la société en groupes statutaires, qui s'accompagne non seulement d'une hiérarchisation des droits, des capacités, mais aussi d'une répartition des types de rapports sociaux, des types de pratiques sociales autorisés ou prescrits, aux uns et aux autres.

Nous l'avons souligné à plusieurs reprises, si les sources écrites présentent des similitudes troublantes dans l'exposition des évolutions sociales judéennes et athéniennes, notre connaissance des réalités sociales judéennes des $\mathrm{VI}^{\mathrm{e}}-\mathrm{IV}^{\mathrm{e}} \mathrm{s}$. reste incertaine, controversée, et les normes et pratiques y paraissent en fait bien plus variées qu'à Athènes. C'est bien sûr un point essentiel. Les sources bibliques témoignent clairement du fait que les régions où se développa la religion juive, avant comme après Esdras, furent caractérisées par des intermariages constants entre populations aux habitudes de vie et aux caractéristiques linguistiques et culturelles très proches. Les mariages avec des esclaves continuèrent également à être pratiqués (cf. Porten 1968, à propos de la communauté d'Éléphantine).

Aux temps d'Esdras et de Néhémie, les informations apportées par les récits de leurs actions démontrent que ces mariages étaient pratiqués par l'ensemble de la population, des prêtres aux membres des clans les plus ordinaires, et que leur interdiction ne fut pas sans provoquer des dissensions; en outre, si ces mariages peuvent impliquer des populations des régions voisines, la formulation de l'interdit oppose en fait aux « fils de la captivité » les " peuples du pays ", autrement dit, ceux qui étaient revenus d'exil d'un côté, et ceux que ces derniers avaient trouvé sur place à leur retour, sans aucun doute, pour une grande part, des descendants de la population judéenne du début du VI $\mathrm{I}^{\mathrm{e}} \mathrm{s}$. Il est donc clair que le véritable enjeu de ces réformes est de définir qui sont les véritables Judéens et dans un même mouvement d'affirmer une pleine autorité sur la communauté, sur sa définition et sur ses comportements.

L'interdiction portant sur toute union mixte mentionnée dans les Livres d'Esdras et de Néhémie est ainsi une injonction qui s'inscrit dans des conflits politiques, religieux et sociaux récurrents. Ces conflits portent non seulement sur la définition de l'identité judéenne, sur les liens sociaux que doivent entretenir les Judéens entre eux et sur la place à accorder aux conversions, mais aussi sur la légitimité des différents pouvoirs politiques et religieux. La récurrence de ce thème des mariages mixtes dans les sources juives de la période du Second Temple témoigne bien d'une absence de consensus au sein de la population (voir notamment Smith-Christopher 1994 ; Rappaport 1996).

En ce qui concerne Athènes, les sources nous donnent quelques informations sur les débats qui ont pu avoir lieu en 403, lors du rétablissement des institutions démocratiques, 
mais si des critères de citoyenneté tels que la propriété foncière ont pu être rejetés, le renouvellement de la loi de Périclès et son application tout au long $\mathrm{du} \mathrm{IV}^{\mathrm{e}} \mathrm{s}$. semblent s'être effectués de manière consensuelle. populations locales est qu'ils se marient entre eux. De ce point de vue, il faut souligner l'un des apports de la théorie anthropologique de l'alliance, développée par Lévi-Strauss, à savoir que les mariages, tout autant que la filiation, définissent et redéfinissent constamment les groupes, leurs contours, leurs compositions.

Nous pouvons reprendre l'exemple des analyses développées par Enric Porqueres (1995) dans une étude consacrée aux Xuetes (les juifs convertis) de Majorque, du XV au XVII ${ }^{\mathrm{e}} \mathrm{s}$. Ainsi qu'il a pu le démontrer, ceux qui, à la fin du XVII ${ }^{\mathrm{e}} \mathrm{s}$., étaient identifiés comme Xuetes ne représentaient en fait qu'une partie des descendants de ceux identifiés comme tels au $\mathrm{XV}^{\mathrm{e}} \mathrm{s}$, et au contraire des personnes qui ne descendaient pas, par les hommes, de juifs convertis, étaient pourtant perçus comme tels. Ce ne furent pas les comportements religieux ou les pratiques alimentaires qui constituèrent le critère déterminant, mais les choix matrimoniaux : certains Xuetes avaient à partir $d u \mathrm{XVI}^{\mathrm{e}}$ s. privilégié les mariages entre proches et ainsi formé un groupe préférentiellement endogame, mais dans lequel entraient également, régulièrement, de nouvelles personnes ; ce sont leurs descendants qui ont donc été identifiés deux siècles après comme Xuetes. E. Porqueres $(1995$, p. 79$)$ résume ce processus d'une formule : « Ce n'est pas parce qu'(ils) sont descendants de juifs qu'ils se marient entre eux, ils "sont" descendants de juifs parce qu'ils se marient entre eux. » Insistons donc encore une fois sur l'idée que le mariage n'est pas simplement ce qui permet à un groupe de se reproduire, il est au cœur de toute construction sociale et identitaire, et il est par excellence ce qui permet de construire du commun, de fondre plusieurs groupes en une seule communauté. Les appartenances, soulignons-le, se transmettent non seulement par la filiation mais aussi par le mariage légitime, et ce fait est bien évidemment en étroite relation avec la généralisation de pratiques endogames, familiales et communautaires.

51 2. Ce qui distingue le cas athénien du cas judéen est que, dans le premier cas, la construction communautaire et statutaire est dans le même temps une construction étatique, et que ce sont les institutions athéniennes dans leur ensemble qui définissent les appartenances et contrôlent les mariages. À la différence de la société judéenne, dépourvue d'instances légitimantes consensuelles, c'est la communauté athénienne en tant qu'État qui impose ses normes collectives, peut susciter un consensus et dans ce processus renforcer le caractère institué et statutaire du mariage.

Ainsi que nous l'avons déjà relevé, à Athènes et de manière plus générale dans l'ensemble du monde grec des cités, le mariage, en tant que «relation d'alliance juridiquement reconnue » devient ainsi une prérogative civique, un "capacité » propre aux citoyens, portant le nom d'epigamia ou d'enguêsis ; cette capacité, que possède bien sûr tout citoyen au sein de sa propre cité, peut certes être perdue en cas d'atimie, mais peut également être octroyée, collectivement ou individuellement, à ceux avec lesquels une cité souhaite renforcer ses liens ou qu'elle souhaite honorer (cf. récemment Coşkun 2013 et 2014).

Cette capacité matrimoniale, cette prérogative accordée à tout citoyen, lui reconnaît le droit non seulement de perpétuer son oikos en engendrant une descendance légitime, 
mais de manière plus générale lui reconnait tout ce qui d'un point de vue juridique est associé au mariage, l'autorité exercée sur un oikos et sur l'épouse qui lui a été accordée (transfert de kureia), la gestion de la dot, l'entrée dans le groupe d'héritage du conjoint ( anchisteia)... C'est désormais dans un cadre institutionnel, donc sous le contrôle constant de la communauté, que se définissent, que s'obtiennent, les statuts parentaux d'époux ou d'épouse, de père ou de mère.

\section{En guise de conclusion} catégories.

Soulignons-le encore une fois, le point de départ de ce trop bref article a donc été la lecture des travaux de chercheurs tels que Saul M. Olyan ou Lisbeth S. Fried qui, en proposant des interprétations fort stimulantes des évolutions connues par la société judéenne au milieu du premier millénaire avant J.-C., se réfèrent plus ou moins explicitement au monde grec qui forme, de fait, un bon point de comparaison. Que faire de ces similitudes et des grilles de lecture proposées? C'est une synthèse personnelle que nous proposons ici et dont il reste à tirer quelques conclusions, encore provisoires.

Les sociétés de Méditerranée orientale, à l'époque classique, témoignent de normes et de pratiques matrimoniales, ou plus généralement parentales, bien évidemment diversifiées: elles peuvent ou non légitimer des unions avec des étrangers, avec des esclaves, avec une seule ou plusieurs épouses, privilégier l'isogamie comme l'anisogamie...; de même, les modes de filiation, de transmission des appartenances, peuvent tout autant présenter des variations importantes (de la patrilinéarité à la matrilinéarité en passant par la bilatéralité).

Est-il possible néanmoins de distinguer au sein de ces variations et des évolutions constatées, des modèles socio-politiques types permettant de penser ensemble les modes de constructions identitaires et étatiques, les rapports de genre, les rapports de parenté, les rapports statutaires, les rapports de subordination et de dépendance, et donc d'englober dans une même réflexion les mariages interdits ou au contraire permis avec des étrangers mais aussi des esclaves?

L'intérêt de la comparaison des évolutions athéniennes et judéennes (pour autant que nos sources nous permettent de les connaître véritablement) pourrait être précisément de mettre en évidence un modèle de construction communautaire (qui ne serait donc pas propre à Athènes), tout à la fois ethnique et statutaire, reposant sur la généralisation, l'universalisation de qualités, normes et pratiques parentales à l'ensemble des membres de la communauté. Dans le cadre de ce modèle, les unions matrimoniales affirmeraient ainsi l'égalité des membres de la communauté et constitueraient l'un des éléments les distinguant radicalement du reste de la population. C'est bien par le contrôle des mariages et de la sexualité que les différences et hiérarchies sociales (ainsi "naturalisées") se transforment et se pérennisent, impliquant de nouveaux rapports sociaux entre

D'autres modèles-types sont bien sûr envisageables, dissociant l'ethnique du statutaire et où le mariage cette fois-ci jouerait un rôle intégrateur, unissant des partenaires d'origines et de statuts différents, dans des relations davantage hiérarchisées; les distinctions statutaires n'y seraient plus radicales, comme dans le cas athénien, mais beaucoup plus graduelles, et donc le passage d'un statut à un autre beaucoup plus aisé, moins soumis à 
un contrôle collectif ; d'autres critères pourraient alors jouer un rôle tout aussi important que la parenté pour hiérarchiser groupes et individus.

De manière schématique, deux modèles peuvent donc être opposés ${ }^{10}$, ainsi que le propose le tableau suivant, modèles bien sûr trop sommaires pour constituer un grille de lecture unique des sociétés méditerranéennes antiques, mais qui permettent néanmoins d'insister sur le rôle spécifique joué par ces opérateurs essentiels d'agrégation et exclusion que sont les normes et pratiques parentales. Les évolutions sociales ainsi constatées sont indissociables du cadre politique dans lequel elles prennent place, et de la manière dont la communauté se construit, se définit.

\begin{tabular}{|l|l|}
\hline Construction ethnique et statutaire & Dissociation de l'ethnique et du statutaire \\
\hline Isogamie & Anisogamie \\
\hline Distinction par le mariage & Intégration par le mariage \\
\hline Distinctions statutaires radicales & Passages plus aisés d'un statut à l'autre \\
\hline Rôle prédominant des critères parentaux & Multiplicité des critères \\
\hline
\end{tabular}

\section{BIBLIOGRAPHIE}

Blok J. H. (2009), « Perikles' Citizenship Law: A New Perspective », Historia 58-2, p. 141-170.

Bonnard J.-B., Dasen V. et Wilgaux J. (2017), Famille et société dans le monde grec et en Italie du Ve au IV siècle av. J.-C., Rennes.

Bonte P. (2000), « Les lois du genre. Approche comparative des systèmes de parenté arabes et touaregs ", dans J.-L. Jamard, E. Terray et M. Xanthakou éd., En substances. Textes pour Françoise Héritier, Paris p. 135-156.

- dir. (1994), Épouser au plus proche. Inceste, prohibitions et stratégies matrimoniales autour de la Méditerranée, Paris.

- et al. dir. (2011), L'argument de la filiation. Aux fondements des sociétés européennes et méditerranéennes, Paris.

Briant P. (1996), Histoire de l'Empire perse, de Cyrus à Alexandre, Paris.

Brulé P. (2007), La Grèce d'à côté. Réel et imaginaire en miroir en Grèce antique, Rennes.

Carawan E. (2008), « Pericles the Younger and the Citizenship Law », CJ 103-4, p. 383-406.

Coşkun A. (2013), « Weitere Überlegungen zu den Voraussetzungen und Folgen des Perikleischen Bürgerrechtsgesetzes : Naturalisierung und Epigamie im Klassischen Athen », Klio 95-2, p. 391404.

- (2014), « Perikles und die Definition des Bürgerrechts im klassischen Athen », HZ 299, p. 1-35. 
Eskenazi T. C. (2006), « The missions of Ezra and Nehemiah », dans Lipschits O. et Oeming M. éd., Judah and the Judeans in the Persian Period, Winona Lake, Indiana, p. 509-530.

Fried L. S. (2009), "The concept of "impure birth" in $5^{\text {th }}$ Century Athens and Judae ", dans Beal R.H., Holloway S. et Scurlock J. éds., In the Wake of Tivka Frymer-Kensky. Tikva Frymer-Kensky Memorial Volume, Piscataway, p. 121-142.

- (2015), « No King in Judah? Mass Divorce in Judah and in Athens », dans Silverman J.M. et Waerzeggers C. éd., Political Memory in and after the Persian Empire, Buenos Aires, p. 381-402.

Fröhlich I. (2005), « Mamzer in Qumran texts: the problem of mixed marriages from Ezra's time. Law, literature and practice », Transeuphratène 29, p. 103-115.

Grabbe L. L. (2004), A History of the Jews and Judaism in the Second Temple Period, vol.1. A History of the Persian Province of Judah, Londres.

Halpern B. (2004), « Ezra's reform and bilateral citizenship in Athens and the Mediterranean world », dans Knoppers Gary N. et Kirsch A. éd., Egypt, Israel and the Ancient Mediterranean World. Studies in honor of Donald B. Redford, Leiden-Boston, p. 439-453.

Hannick J. M. (1976), « Droit de cité et mariages mixtes dans la Grèce classique. À propos de quelques textes d'Aristote (Pol. 1275b, 1278a, 1319b) », AC XLV, p. 133-148.

Lape S. (2002-2003), « Solon and the institution of the democratic family form », CJ 98, p. 117-139. Leduc Cl. (1990), « Comment la donner en mariage ? ", dans Duby G. et Perrot M. dir., Histoire des femmes en Occident, t. I : Antiquité, Schmitt Pantel P. éd., Paris, p. 259-315.

Lemaire A. (2015), « Esclavage pour dettes et autres formes de travail dépendant au Levant: tradition biblique et épigraphie ( $X^{\mathrm{e}}-\mathrm{V}^{\mathrm{e}} \mathrm{s}$.) », dans Zurbach J. éd. (2015), p. 67-84.

Lemos T. M. (2010), Marriage Gifts and Social Change in Ancient Palestine. 1200 BC to 200 CE, Cambridge.

Mactoux M.-M. (1988), « Lois de Solon sur les esclaves et formation d'une société esclavagiste », dans Forms of Control and Subordination in Antiquity, Leiden, p. 331-354.

Olyan S. M. (2000), Rites and Rank. Hierarchy in Biblical Representations of Cult, Princeton.

Porqueres i Gené E. (1995), Lourde alliance. Mariage et identité chez les descendants de Juifs convertis à Majorque (1435-1750), Paris.

Porten B. (1968), Archives from Elephantine. The life of an ancient jewish military colony, BerkeleyLos Angeles.

Rappaport U. (1996), « Les Juifs et leurs voisins à l'époque perse, hellénistique et romaine », Annales (HSS) 51-5, p. 955-974.

Roussel P. (1976), Tribu et cité, Besançon-Paris.

Satlow M.L. (2001), Jewish Marriage in Antiquity, Princeton.

Smith-Christopher D. (1994), « The mixed marriage crisis in Ezra 9-10 and Nehemiah 13: a study of the sociology of post-exilic Judaean community ", dans Eskenazi T.C. et Richards K.H. éd., Second Temple Studies 2. Temple Community in the Persian Period, Sheffield, p. 243-265.

Vélissaropoulos-Karakostas J. (2011), Droit grec d'Alexandre à Auguste, 323 av. J-C. - 14 ap. J.-C. : personnes, biens, justice, Athènes.

Vérilhac A.-M. et Vial Cl. (1998), Le mariage grec du VI siècle avant J.-C. à l'époque d'Auguste, Paris.

Walters K.R. (1983), «Pericles'citizenship law », ClAnt 2, p. 314-336. 
Wilgaux J. (2011), « Les groupes de parenté en Grèce ancienne : l'exemple athénien », dans Bonte P., Porqueres i Gené E. et Wilgaux J. éd., L'argument de la filiation. Aux fondements des sociétés européennes et méditerranéennes, Paris, p. 327-348.

Zurbach J. (2013), « La formation des cités grecques. Statuts, classes et systèmes fonciers », Annales (HSS) 68-4, p. 957-998.

- éd. (2015), La main-d'œuvre agricole en Méditerranée archaïque. Statuts et dynamiques économiques, Bordeaux.

\section{NOTES}

1. Mes remerciements les plus vifs vont aux organisateurs du colloque ainsi qu'à tous ceux qui lors de ces journées, par leurs interventions, m'ont permis de compléter les analyses développées dans cet article.

2. Le caractère institué de la parenté apparaît clairement dans les sources lexicographiques grecques, cf. par ex. Harpocration et la Souda, s.u. gennêtai.

3. Cf. Politiques, III, 2, 1275b21-22 (trad. Tricot remaniée) : «Selon l'usage courant, un citoyen se définit comme l'enfant né de parents tous deux citoyens, et non pas d'un seul, son père ou sa mère. »

4. Cf. Hannick 1976; Vérilhac et Vial 1998, p. 43 sq.; Vélissaropoulos-Karakostas 2011, p. 210 sq. ; Bonnard et al. 2017, p. 262 sq.

5. Et ce fut d'ailleurs peut-être le cas à Athènes avant Périclès, d'où la difficulté d'interpréter la loi de Périclès comme une meilleure reconnaissance, une valorisation du rôle des Athéniennes.

6. Soulignons ainsi que les évolutions politiques, sociales, économiques du monde grec ainsi que les modalités de construction des cadres civiques grecs ne peuvent être dissociées du cadre méditerranéen dans lesquelles elles trouvent place, cf. notamment Zurbach 2013 et 2015. Nous qualifions donc de « mariage mixte » un mariage réalisé avec un partenaire extérieur à la «communauté » : cette dernière peut être diversement définie, mais ainsi que les travaux que nous reprenons ici tentent de le montrer, les modes de construction des communautés grecques et judéenne ne sont pas sans présenter des similitudes, et accordent une place essentielle aux normes matrimoniales : tel est l'objet de cette étude.

7. Ce thème de l'interdit des mariages mixtes apparaît en fait comme un leitmotiv des écrits de la période du Second Temple et des sources postérieures aux livres d'Esdras et Néhémie, tel que le Livre des Jubilés (écrit sans doute au II $\mathrm{s}$. av. J.-C.), jugent encore plus sévèrement les mariages avec des étrangers (voir notamment 30,7) et définissent le Judéen par la pratique de la circoncision; tout mariage avec un incirconcis devient donc interdit pour une Judéenne.

8. Citons, parmi les explications les plus souvent proposées, de l'interdiction des mariages mixtes, à Athènes comme en Judée: la protection de la pureté d'origine de la communauté ; la volonté d'une communauté de maintenir ses traditions, ses privilèges, dans un contexte de présence massive d'« étrangers "; la conservation des patrimoines, notamment fonciers ; la limitation du nombre de « citoyens »; l'interdiction de mariages privilégiés jusqu'alors par les élites... 
9. Les deux sociétés conservent bien sûr des spécificités, avec par exemple dans le cas judéen le mariage léviratique.

10. Voir également les modèles et le cadre interprétatif proposés dans de nombreux travaux par Pierre Bonte, e.g. Bonte 2000.

\section{RÉSUMÉS}

Si la loi de Périclès de 451-450, qui restreignait la citoyenneté athénienne aux seuls fils de deux parents athéniens, a suscité de nombreux commentaires, ces derniers cependant tiennent peu compte du fait que cette exigence d'une double ascendance se retrouve dans bien d'autres cités du monde grec ainsi que dans d'autres sociétés méditerranéennes. Cet article se propose ainsi de comparer le cas grec au cas judéen, marqué par l'interdiction répétée, à l'époque classique, des mariages mixtes, à l'initiative d'Esdras et de Néhémie. Il parait alors possible d'associer à ce choix matrimonial commun d'autres similitudes dans le modèle de construction communautaire adopté par ces deux sociétés, avec notamment une réorganisation progressive des institutions familiales. C'est ainsi dans un même mouvement que les constructions identitaires et statutaires renouvellent les rapports sociaux et économiques au sein de ces deux sociétés.

Although Pericles' citizenship law of 451-450, which restricted Athenian citizenship to the sons of two Athenian parents, has given rise to many studies, these comments, however, take little into account the fact that this requirement of a double ancestry is found in other cities of the Greek world as well as in other Mediterranean societies. This article attempts to compare the Greek case with the Judean case, which is marked by the prohibition of mixed marriages in the classical period, on the initiative of Ezra and Nehemiah. It seems then possible to associate with this common matrimonial choice other similarities in the model of community construction adopted by these two societies, notably a progressive reorganization of family institutions. It is thus in the same movement that the constructions of identity and status renew the social and economic relations within these two societies.

\section{INDEX}

Keywords : kinship, marriage, citizenship, Judah, Athens

Mots-clés : parenté, mariage, citoyenneté, Judée, Athènes

\section{AUTEUR}

\section{JÉRÔME WILGAUX}

Université de Nantes, CRHIA (Centre de Recherche en Histoire Internationale et Atlantique) 